\title{
Raman and Normal-Mode Studies of the Extended-Helix Conformation in Polypeptide Chains*
}

\author{
P. K. SENGUPTA ${ }^{\dagger}$ and S. KRIMM, Biophysics Research Division \\ and Department of Physics, University of Michigan, Ann Arbor, \\ Michigan 48109
}

\begin{abstract}
Synopsis
The nature of the local main-chain conformation of polypeptides with charged side chains has been the subject of considerable discussion since Tiffany and Krimm first proposed [(1968) Biopolymers 6, 1379-1381] that, rather than being random, the chain is locally relatively regular, with conformations similar to that of a left-handed threefold helix. Such structures, referred to as "extended-helix" (EH) conformations, have now been studied in a charged poly(L-glutamic acid) system by a combination of Raman spectroscopy and normal-mode analysis. Calculations were done for EH conformations with 3.0 and 2.4 residues/turn, using force fields refined for $\alpha$-helix, $3_{1}$-helix, and $\beta$-sheet structures. Together with previous results on the $\alpha$-helix and $\beta$-sheet forms, an interesting new correlation emerged: the frequency of the $\mathrm{C}^{\alpha} \mathrm{C}$ stretch skeletal mode, usually found in the $900-1000 \mathrm{~cm}^{-1}$ region of the Raman spectrum, is essentially linearly correlated with the value of the $\phi$ angle. Applying this relationship to the observed frequencies of the $\alpha$-helix and $\beta$-sheet forms of poly(L-glutamic acid), we find that an observed sharp band in the spectrum of crystals of the calcium salt of poly(L-glutamic acid) (which is close to the frequency observed for the charged form in solution) corresponds to an EH conformation very clase to that predicted from energy calculations. These studies thus provide very strong support for our proposal that charged polypeptide chains are not random but adopt local conformations of the EH type.
\end{abstract}

\section{INTRODUCTION}

On the basis of $\mathrm{CD}$ studies on poly(L-glutamic acid) $\left[(\mathrm{GluH})_{n}\right]$ and poly(Llysine) $\left[(\mathrm{Lys})_{n}\right]$, Tiffany and Krimm ${ }^{1}$ proposed in 1968 that the local conformation of polypeptides having charged side chains is not random, as was previously thought, but consists of regions of relative order in which the chain adopts conformations similar to that of a left-handed $3_{1}$-helix. Simplified conformational energy calculations ${ }^{2}$ indicated that such a charged helix should actually have about 2.5 residues/turn, and these structures were referred to as "extended-helix" (EH) conformations. ${ }^{2}$ Locally random conformations, with CD spectra quite different from those of the $\mathrm{EH}$ form, could be obtained under a variety of conditions, including aqueous salt solution ${ }^{3}$ and heat denaturation, ${ }^{4}$ and on a variety of systems, including synthetic polypeptides, fibrous proteins, and globular proteins. ${ }^{4}$ The predictions of a theo-

\footnotetext{
* This is paper number 36 in a series on vibrational analysis of peptides, polypeptides, and proteins, of which Ref. 23 is paper number 35.

${ }^{\dagger}$ Present address: Saha Institute of Nuclear Physics, Calcutta 70064, India.
} 
retical study ${ }^{5}$ were consistent with the proposed assignment of these $\mathrm{CD}$ spectra to such a locally disordered chain.

The results of other CD studies under different environmental conditions ${ }^{6-8}$ have strongly supported the above interpretations. In addition, an assessment of the overall evidence ${ }^{9}$ has indicated that the objections raised to these views are not convincing, and that the originally proposed CD spectral and structural assignments remain valid. This conclusion has received continuing support from subsequent $\mathrm{CD}$ studies. ${ }^{10-13}$

Since the EH conformation is not restricted to solubilized polypeptides with charged side chains, ${ }^{7-10}$ and has even been found by $\mathrm{x}$-ray diffraction analysis in a crystalline globular protein, ${ }^{14}$ it is important to have other methods for characterizing this structure. Vibrational spectroscopy provides such a technique, and in fact, Raman spectra have been used to investigate the structure of charged polypeptide chains. Koenig and Frushour ${ }^{15}$ studied the Raman spectra of $(\mathrm{GluH})_{n}$ as a function of $\mathrm{pH}$; in addition to intensity changes in the amide III region, they noted a large frequency shift in the skeletal stretch region, from $931 \mathrm{~cm}^{-1}$ at low $\mathrm{pH}$ ( $\alpha$-helix form) to $949 \mathrm{~cm}^{-1}$ at high $\mathrm{pH}$ (charged form). Yu et al ${ }^{16}$ did similar studies on (Lys) ${ }_{n}$; they noted that, in the skeletal stretch region, the frequency for the charged form $\left(958 \mathrm{~cm}^{-1}\right)$ is intermediate between those for the $\alpha$-helix $\left(945 \mathrm{~cm}^{-1}\right)$ and the $\beta$-sheet $\left(\sim 1002 \mathrm{~cm}^{-1}\right)$ forms. Painter and Koenig ${ }^{17}$ did a combined Raman and ir study on (Lys) ${ }_{n}$. They presented several arguments in favor of the presence of regions of local order in the charged form: (1) The amide III band in the charged form is relatively sharp, compared to broader bands for disordered proteins. This mirrors a similar change in the amide III band of (AlaGlyGly) ${ }_{n}$, a polypeptide known to form a left-handed $3_{1}$-helix, ${ }^{18,19}$ when it is disordered by heating. ${ }^{11}(2)$ The amide I mode exhibits a significant splitting $\left(23 \mathrm{~cm}^{-1}\right)$ between Raman and ir, much larger than that for the $\alpha$-helix or for disordered $(\mathrm{GluNa})_{n}{ }^{17}$; it is therefore presumably due to an ordered structure. They noted the analogy with the $10 \mathrm{~cm}^{-1}$ splitting in poly(glycine II) [(Gly) $\left.{ }_{n} \mathrm{II}\right]$, which has a left-handed $3_{1}$-helix structure, and for which the splitting has now been reproduced by a normal-mode calculation..$^{20}$ (3) The amide III band of (Lys) ${ }_{n}$ in $4 M \mathrm{CaCl}_{2}$, which destroys local order, ${ }^{4}$ is similar to that of (DL-Lys) ${ }_{n}$ in $\mathrm{H}_{2} \mathrm{O}$, a polypeptide thought to be substantially disordered. Sugawara et al..$^{2 \mathrm{O}}$ obtained preresonance Raman spectra of the charged forms of (GluH $)_{n}$ and (Lys) ${ }_{n}$, and concluded that their results could be explained by the presence of some ordered structures [their amide I frequency for (Lys) resulted in a splitting of $16 \mathrm{~cm}^{-1}$ between Raman and ir modes].

Although such Raman studies provide important structural insights, they cannot carry the conviction possible through the rigorous treatment provided by normal-mode analysis. ${ }^{22}$ Such calculations permit definitive interpretations of spectra in terms of three-dimensional structure, as well as a detailed understanding of the dynamics of these structures. ${ }^{23}$ We have therefore attempted to analyze the normal modes of the $\mathrm{EH}$ form of $\left(\mathrm{Glu}^{-}\right)_{n}$, and to relate these predictions to experimental results on this polypeptide. We have chosen this system because of our prior normal-mode studies of the $\beta$-sheet ${ }^{24}$ and $\alpha$-helix ${ }^{25}$ forms, as well as our experimental studies on the $\mathrm{Mg}^{2+}$ and $\mathrm{Ca}^{2+}$ salts of this molecule. ${ }^{26} \mathrm{As}$ we will see, analysis of the skeletal stretching region provides convincing evidence of the existence of the $\mathrm{EH}$ conformation. 


\section{EXPERIMENTAL}

Lamellar hexagonal crystals of (GluCa) ${ }_{n}$ were obtained by following the procedure outlined by Keith. ${ }^{27}$ The samples used in the present study are the same as reported in our earlier communication. ${ }^{26}$

Raman spectra were recorded on a Spex 1403 spectrometer with a Datamate computer, using the $5145 \AA$ exciting line of a Spectra Physics $165 \mathrm{Ar}^{+}$ laser. For recording Raman spectra of crystals in the wet state, the crystals were sealed in glass capillaries as slurries in ethanol-water mixtures.

\section{NORMAL-MODE CALCULATIONS}

Two EH structures were used in the normal-mode calculations: (1) A left-handed $3_{1}$-helix, i.e., $n=-3.0$, with a helix axis repeat per residue of $h=3.1 \AA$; this is the same backbone structure as that of $(\mathrm{Gly})_{n} \mathrm{II}^{20}$ (2) A left-handed helix with $n=-2.4$ and $h=3.2 \AA$; this corresponds to a minimum-energy conformation for $\left(\mathrm{Glu}^{-}\right)_{n}$ obtained from more complete calculations. ${ }^{28}$

As in our previous calculations, ${ }^{24,25}$ the $\mathrm{COO}^{-}$-terminated side chains were assumed to be fully extended. This is a reasonable assumption in terms of minimizing the electrostatic energy. ${ }^{2}$ Bond lengths and angles for the peptide backbone as well as for the side chain were the same as used previously. ${ }^{24,25}$ Since the solvent $\left(\mathrm{H}_{2} \mathrm{O}\right)$ interacts with the backbone by hydrogen bonding to the peptide group, we have placed $\mathrm{H}$ and $\mathrm{O}$ atoms in appropriate positions with respect to the $\mathrm{C}=0$ and $\mathrm{NH}$ groups, respectively, using the same hydrogen-bond geometry as in $\beta$-(GluCa) ${ }_{n} \cdot{ }^{24}$ For our present purposes, this is a satisfactory assumption. The definitions of internal and local symmetry coordinates followed our previous work. ${ }^{24,25}$

The force field used in the calculations is not obvious. Unlike the previous calculations of $\beta$-(GluCa) ${ }_{n}{ }^{24}$ and $\alpha$-(GluH $)_{n},{ }^{25}$ where existing force fields for $\beta$-(Ala) ${ }_{n}{ }^{29,30}$ and $\alpha$-(Ala) ${ }_{n}{ }^{31}$ could be used without further refinement, there is at this time no refined force field for single polypeptide chains having $\mathrm{EH}$ conformations. It might be though that the force field of (Gly) ${ }_{n}$ II would be most suitable, but the presence of a $\mathrm{CH}_{2}$ group in the chain has a significant effect on the frequencies. ${ }^{22}$ In the absence of a clear-cut optimum force field, we have adopted the following approach: For structures 1 and 2, calculations were done using the force fields for $\beta$-(Ala $)_{n},{ }^{29,30}(\mathrm{Gly})_{n} \mathrm{II},{ }^{20}$ and $\alpha$-(Ala $)_{n}{ }^{31}$ Together with the results for $\beta$-(GluCa) ${ }_{n}{ }^{24}$ and $\alpha$-(GluH $)_{n},{ }^{25}$ these calculations can show which normal modes are particularly sensitive to the polypeptide backbone conformation, and the nature of such a dependence on structure. As indicated above, we find a significant effect of this kind in the skeletal stretch region, and we will therefore concentrate on one of these normal modes. There are no such major effects on higher frequency modes, and although some lower frequency skeletal modes do depend on backbone chain conformation, they are not associated with strong characteristic observed bands.

\section{RESULTS AND DISCUSSION}

In Fig. 1(a) we present the Raman spectrum in the $900-1000 \mathrm{~cm}^{-1}$ region of crystalline (hexagonal) $(\mathrm{GluCa})_{n}$ in the wet state. The crystals were in the 


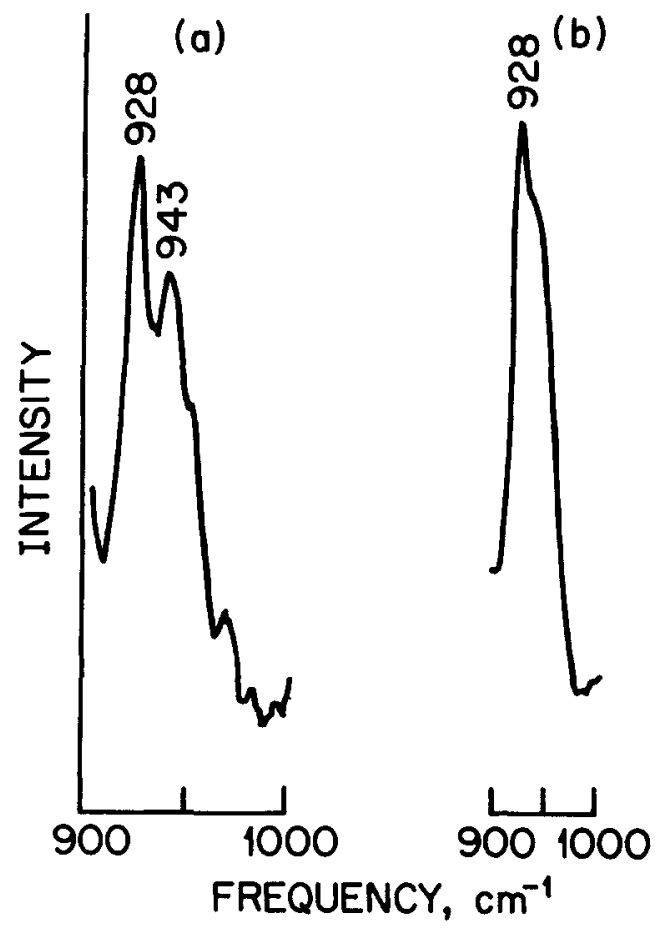

Fig. 1. Raman spectra of crystalline (hexagonal) (GluCa) $)_{n}$ (a) In the wet state (a slurry of crystals in $10 \%$ ethanol-water). (b) In the dry state. Laser power $60 \mathrm{~mW}$, spectral slit width $\sim 5.5$ $\mathrm{cm}^{-1}$

form of a slurry in a $10 \%$ ethanol-water mixture. A very strong band is seen at $928 \mathrm{~cm}^{-1,26}$ with a well-defined strong companion band at $943 \mathrm{~cm}^{-1}$. When the crystals are dried, ${ }^{26}$ the latter band is reduced to a shoulder [cf. Fig. 1(b)]. We also found a spectrum (not shown) very similar to Fig. 1(b) when the crystals were suspended in $100 \%$ ethanol.

Strong Raman bands in this region are associated with a skeletal stretch mode that is predominantly $\mathrm{C}^{\alpha} \mathrm{C}$ stretch (s) combined with $\mathrm{CN} s$ and $\mathrm{CO} \mathrm{s}^{22}$ [In (Gly) ${ }_{n} \mathrm{I}$ and (Gly) $)_{n} \mathrm{II}$, this mode is found at $884 \mathrm{~cm}^{-1}$, but in these cases it also has a $\mathrm{CH}_{2}$ rock (r) component.] It might seem that this mode is insensitive to chain conformation, since analogous modes are found at about the same frequency $\left(\sim 908 \mathrm{~cm}^{-1}\right)$ for $\beta-(\mathrm{Ala})_{n}$ and $\alpha-(\mathrm{Ala})_{n}{ }^{22}$ However, this is not the case for polypeptide chains with side chains longer than $\mathrm{CH}_{3}$ : this mode is observed at $956 \mathrm{~cm}^{-1}$ in $\beta$-(GluCa $)_{n}^{24}$ and at $924 \mathrm{~cm}^{-1}$ in $\alpha-(\mathrm{GluH})_{n},{ }^{25}$ and at $\sim 1002$ and $945 \mathrm{~cm}^{-1}$ for the corresponding forms of (Lys) ${ }_{n}{ }^{16}$ (These data indicate a dependence of the frequency in a given form on the length of the side chain. ${ }^{22}$ ) It is therefore reasonable to associate the two bands at 928 and $943 \mathrm{~cm}^{-1}$ in the wet (GluCa) ${ }_{n}$ crystals with two different chain conformations. The $928 \mathrm{~cm}^{-1}$ band is clearly correlated with the $\alpha$-helix form, ${ }^{25,26}$ an assignment supported by x-ray diffraction studies on (GluMg) ${ }_{n} \cdot{ }^{27,32}$ The 943 $\mathrm{cm}^{-1}$ band, which, interestingly, is close to the frequency of $-948 \mathrm{~cm}^{-1}$ found for $\left(\mathrm{Glu}^{-}\right)_{n}$ in solution, ${ }^{15,21}$ could well be associated with an EH conformation, particularly in view of the indirect evidence from x-ray diffrac- 
TABLE I

Dependence of Calculated Frequencies (in $\mathrm{cm}^{-1}$ ) on Chain Conformation for the A-Species Skeletal Stretch Mode of Charged Poly(L-Glutamic Acid)

\begin{tabular}{|c|c|c|c|c|c|c|c|}
\hline \multirow{5}{*}{$\begin{array}{l}\text { Force } \\
\text { Field }^{\mathrm{a}}\end{array}$} & \multicolumn{4}{|c|}{ Structure $^{\mathrm{b}}$} & \multicolumn{3}{|c|}{$\mathrm{PED}^{\mathrm{c}}$} \\
\hline & $2_{1}$ & $2.4_{1}$ & $3_{1}$ & $3.62_{1}$ & & & \\
\hline & -134.84 & $\overline{-96.14}$ & $\overline{-79.28}$ & -57.37 & & & \\
\hline & 132.01 & 129.38 & 150.50 & -47.49 & & & \\
\hline & 3.415 & 3.20 & 3.10 & 1.495 & $\mathrm{C}^{\alpha} \mathrm{Cs}$ & $\mathrm{CN} \mathrm{s}$ & $\mathrm{COs}$ \\
\hline \multirow[t]{3}{*}{$\beta$} & 944 & & & & 18 & 13 & 11 \\
\hline & & 928 & & & 23 & 14 & 12 \\
\hline & & & 923 & & 25 & 15 & 11 \\
\hline \multirow[t]{2}{*}{ PGII } & & 938 & & & 24 & 12 & 13 \\
\hline & & & 930 & & 26 & 12 & 13 \\
\hline \multirow[t]{3}{*}{$\alpha$} & & 956 & & & 13 & 12 & 9 \\
\hline & & & 946 & & 17 & 14 & 12 \\
\hline & & & & 922 & 9 & 27 & 9 \\
\hline
\end{tabular}

${ }^{\mathrm{a}} \beta$ : from $\beta$-(Ala $)_{n}{ }^{29}$; PGII: from $(\mathrm{Gly})_{n} \mathrm{II}^{20} ; \alpha$ : from $\alpha-(\mathrm{Ala})_{n}{ }^{30}$.

${ }^{b}$ The three numbers under each structure correspond to $\phi, \psi$ (both in degrees), and $h$ (in $\AA$ ). $2_{1} \equiv \beta$-sheet; $2.4_{1}, 3_{1} \equiv$ EH conformations; $3.62_{1} \equiv \alpha$-helix.

${ }^{\mathrm{c}}$ Potential energy distribution for backbone stretch coordinates.

tion for such a structure. ${ }^{27}$ More definitive evidence for such a proposal comes from the results of the normal-mode calculations.

In Table I we present the calculated frequencies of the A-species skeletal stretch modes in the $900-1000 \mathrm{~cm}^{-1}$ region (corresponding to the observed strong Raman band) for the conformations under consideration. The results for the $2.4_{1}{ }^{-}$and $33^{-}$-helices are from the present study; those for the $2_{1}{ }^{-}$and $3.62_{1}$-structures are from earlier calculations on $\beta$-(GluCa ${ }_{n}{ }^{24}$ and $\alpha-(\mathrm{GluH})_{n},{ }^{25}$ respectively. The dihedral angles $\phi$ (torsion about $\mathrm{NC}^{\alpha}$ ) and $\psi$ (torsion about $\mathrm{C}^{\alpha} \mathrm{C}$ ), as well as $h$, are also given for each structure. The potential energy distribution (PED) for this mode is quite complex, but in the table we give only the contributions from the backbone stretch coordinates. [For all structures other than the $\alpha$-helix, additional contributions in the range of $5-10$, are made to the PED by the following coordinates: $\mathrm{C}^{\beta} \mathrm{C}^{\gamma} \mathrm{s}, \mathrm{C}^{\gamma} \mathrm{C}^{\delta} \mathrm{s}, \mathrm{NC}^{\alpha} \mathrm{C}$ deformation (d), $\mathrm{C}^{\alpha} \mathrm{CN} \mathrm{d}$, and $\mathrm{CNC}^{\alpha} \mathrm{d}$. For the $\alpha$-helix, the additional contributions are from $\mathrm{CNC}^{\alpha} \mathrm{d}$ and $\mathrm{CO}$ in-plane bend.]

What is immediately apparent from Table $I$ is that, independent of the force field, this skeletal stretch frequency decreases as the "extension" of the chain backbone decreases (cf. the frequencies as a function of $h$ ). (This was already noted in studies using earlier versions of our force fields. ${ }^{33}$ ) The reason for this seems associated with the relatively increasing proportion of $\mathrm{C}^{\alpha} \mathrm{C} s$ in the mode (compared to $\mathrm{CN} \mathrm{s}$ and $\mathrm{CO} s$ ) as the chain extension decreases. (The $\alpha$-helix structure is an exception to this trend, perhaps because of its different, viz., internal, hydrogen-bonding topology. The $\mathrm{CN}$ s contribution is also unusually large in this case.) Such a correlation must reflect the influence of the local chain conformation, and in fact, as seen from Fig. 2, this skeletal stretch frequency shows a relatively uniform dependence on $\phi$. Whether or not the variation is approximately linear, as is suggested by Fig. 2, cannot be determined at present, since we are not certain of the exact force field 


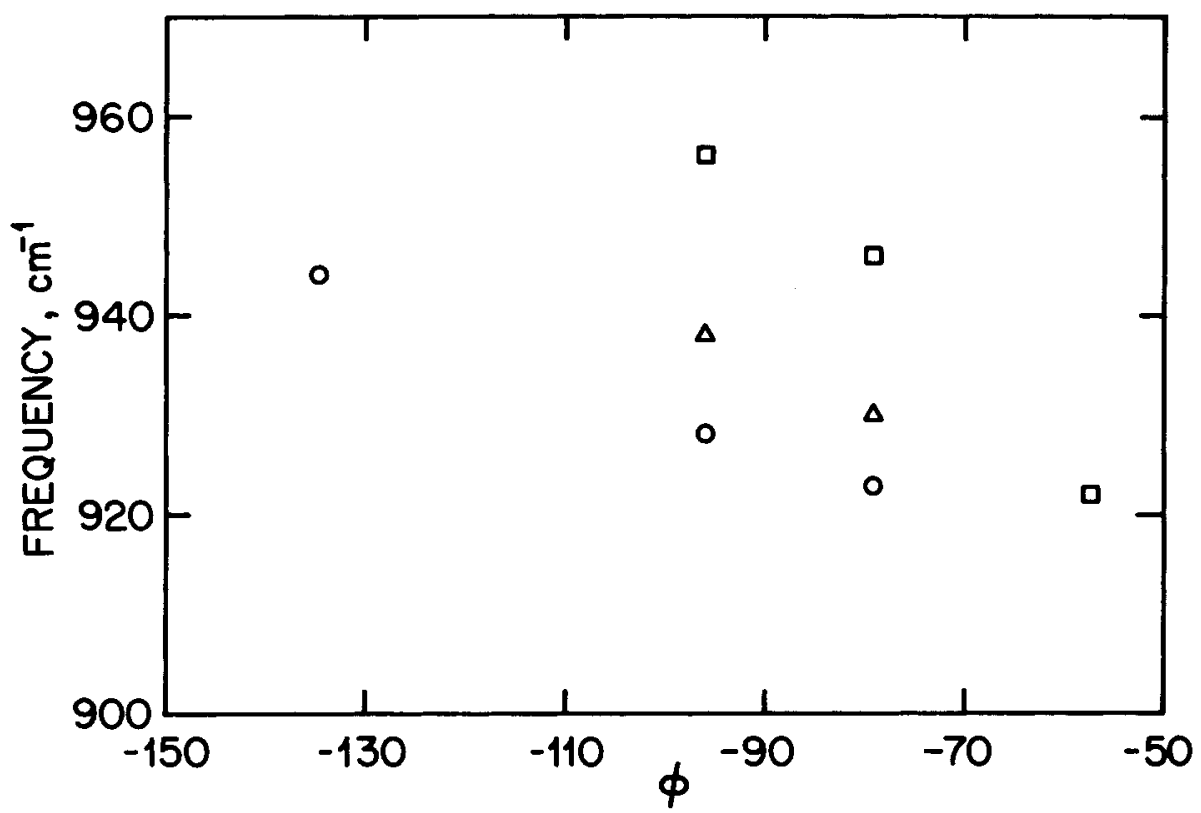

Fig. 2. Variation of calculated A-species $\mathrm{C}^{\alpha} \mathrm{C}$ stretch skeletal frequency with dihedral angle $\phi$. Calculations done with (O) $\beta$ force field, ( $\Delta$ ) polyglycine II force field, (D) $\alpha$-helix force field.

appropriate to the $\mathrm{EH}$ conformations. It is, however, clear from the normalmode analysis that this mode exhibits a specific and systematic sensitivity to backbone conformation.

It is of interest to inquire into possible reasons for a dependence of this frequency on $\phi$ and, as can be seen from Table I, its essential independence of $\psi$. The main reason for the $\phi$ dependence is probably the combined involvement of $\mathrm{C}^{\alpha} \mathrm{C} \mathrm{s}$ and $\mathrm{CN} \mathrm{s}$ in this mode, which is the only mode to which $\mathrm{C}^{\alpha} \mathrm{C} \mathrm{s}$ makes a large contribution. Since $\mathrm{CN} s$ is an important component of the mode, it is possible to see how its contribution could vary with $\phi$ : Considering the two peptide groups that adjoin a given residue (cf. Fig. 3), and the fact that the orientation of the $\mathrm{CN}(1)$ bond with respect to the $\mathrm{C}^{\alpha} \mathrm{C}$ bond changes as $\phi$ changes whereas there is no effect of the $\operatorname{CN}(2)$ bond as a function of $\psi$, it is apparent that the interaction of $\mathrm{CN}(1) \mathrm{s}$ with $\mathrm{C}^{\alpha} \mathrm{C} \mathrm{s}$ through the kinetic energy matrix can vary with $\phi$; that is, the extent of mixing of $\mathrm{C}^{\alpha} \mathrm{C} s$ and $\mathrm{CN} s$

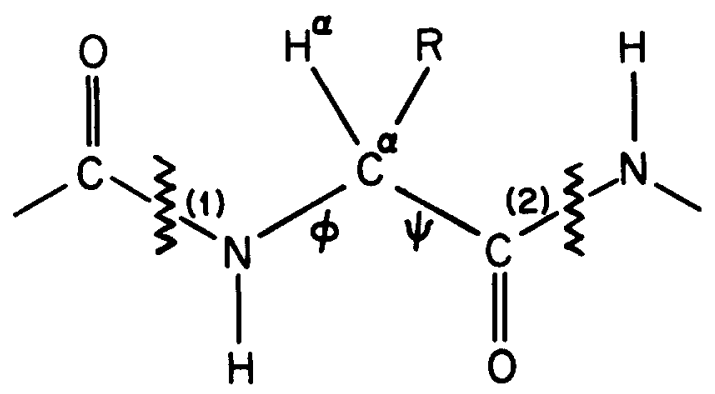

Fig. 3. Portion of polypeptide chain, relating adjacent $\mathrm{CN}$ bonds, (1) and (2), to $\mathrm{C}^{\alpha} \mathrm{C}$ bond. 
should, in principle, be a function of $\phi$. It might be thought that the frequency should also depend on $\psi$ through changes in the relative positions of $\mathrm{C}^{\beta}, \mathrm{H}^{\alpha}$, and $\mathrm{N}$ with respect to the $\mathrm{C}^{\alpha} \mathrm{C}(=0) \mathrm{N}$ group, but there is a good reason to expect this effect to be minimal: Since the eigenvector contains no significant contributions from coordinates associated with these substitutents - such as $\mathrm{NC}^{\alpha} \mathrm{s}, \mathrm{H}^{\alpha}$ bend, and $\mathrm{C}^{\alpha} \mathrm{C}^{\beta} \mathrm{s}$-it is not likely that changes in the positions of the substituents will have a significant influence on the frequency of the mode. Of course, these arguments are specific to the eigenvector for this mode in $\left(\mathrm{Glu}^{-}\right)_{n}$. In $(\mathrm{Gly})_{n}$ and $(\mathrm{Ala})_{n}, \mathrm{CH}_{2} \mathrm{r}$ and $\mathrm{CH}_{3} \mathrm{r}$, respectively, contribute to the PED of this mode, and the coupling between internal coordinates may be different as a function of $\phi$ and $\psi$. Nor can the dependence in (Lys) ${ }_{n}$, for example, be predicted with confidence in the absence of a normal-mode calculation on this structure. What can be said, however, is that the calculations for $\left(\mathrm{Glu}^{-}\right)_{n}$ show that a convincing case can be made for a close-to-linear dependence of this skeletal stretch frequency on $\phi$ over the range of about $-60^{\circ}$ to $-135^{\circ}$.

The above insights provide strong support for the proposal that the 943 $\mathrm{cm}^{-1}$ band of wet (GluCa) ${ }_{n}$ crystals represents a chain conformation intermediate in extension between that of the $\alpha$-helix, represented by the observed band at $928 \mathrm{~cm}^{-1}$, and that of the $\beta$-sheet, represented by an observed band at $956 \mathrm{~cm}^{-1} .{ }^{24}$ If we assume a linear relation between frequency and $\phi$, the observed $943 \mathrm{~cm}^{-1}$ band would correspond to a $\phi$ value of $-98.87^{\circ}$, quite close to the $\phi$ value of $-96.14^{\circ}$ calculated for the minimum-energy EH conformation. ${ }^{28}$ It is interesting that, for the wet (GluCa) ${ }_{n}$ crystals, the half-width (full width at half-maximum) $\Delta \nu_{1 / 2}$ of the $943 \mathrm{~cm}^{-1}$ band is approximately the same $\left(\sim 14 \mathrm{~cm}^{-1}\right)$ as that of the $\alpha$-helix band at $928 \mathrm{~cm}^{-1}$ [cf. Fig. 1(a)], suggesting that these molecules in the crystal (whose relative organization we do not know) have comparable regularity along the chain. When the crystals are dried or suspended in $100 \%$ ethanol, the proportion of the EH structure decreases without any significant increase in $\Delta \nu_{1 / 2}$, a result consistent with the expected influence of the dielectric constant of the solvent on the stability of the EH conformation. ${ }^{6}$ However, for the charged helix in solution, ${ }^{15} \Delta \nu_{1 / 2}$ for the $949 \mathrm{~cm}^{-1}$ band is much larger $\left(\sim 40 \mathrm{~cm}^{-1}\right)$, indicating a broader distribution in $\phi$ under these circumstances. This is entirely ${ }_{t s}$ reasonable, since we suppose the chain in solution to be a coil those "statistical segments" exhibit local order in $\phi, \psi$ corresponding to the EH type of conformation, ${ }^{9}$ and since these segments are of varying length and environment, it would be expected that there would be a larger distribution in $\phi$ than would be the case in the ordered environment of a crystal. Thus, normal-mode analysis of the observed Raman spectra of $\left(\mathrm{Glu}^{-}\right)_{n}$ in crystals and in solution provides definitive evidence to support the early contention ${ }^{1}$ that polypeptide chains with charged, as well as in some cases noncharged, ${ }^{7-10}$ side chains adopt local conformations of the $\mathrm{EH}$ type.

\section{CONCLUSIONS}

Our normal-mode calculations show that the $\mathrm{C}^{\alpha} \mathrm{C}$ s skeletal mode, observed as a strong Raman band in the $900-1000 \mathrm{~cm}^{-1}$ region, is highly sensitive to backbone chain conformation in $\left(\mathrm{Glu}^{-}\right)_{n}$. This is in contrast to its apparent 
independence of such conformational change in the case of polypeptides with shorter side chains, ${ }^{22}$ such as $(\mathrm{Gly})_{n}$ and (Ala) $)_{n}$. The frequency of this mode increases with increasing extension of the backbone, exhibiting a nearly linear increase with decreasing value of the $\phi$ angle. By using the latter relationship, and the observed frequencies of 928 and $956 \mathrm{~cm}^{-1}$ for the $\alpha$-helix and $\beta$-sheet forms, respectively, we find that the observed band at $943 \mathrm{~cm}^{-1}$ in wet $(\mathrm{GluCa})_{n}$ crystals corresponds to an $\mathrm{EH}$ conformation very close to that predicted from energy calculations. ${ }^{28}$ These results thus confirm our early proposal, based on CD studies, ${ }^{1}$ that such a conformation is a preferred one in polypeptides with charged side chains.

The present work suggests that this skeletal mode in $\left(\mathrm{Glu}^{-}\right)_{n}$ can be useful in monitoring variations in the $\mathrm{EH}$ conformation with changes in environment. Thus, if the frequency increase between the crystals, $943 \mathrm{~cm}^{-1}$ [Fig. 1(a)], and the high $\mathrm{pH}$ form in solution, ${ }^{15} 949 \mathrm{~cm}^{-1}$, reflects a change in average conformation (from $\phi=-98.87^{\circ}$ to $\phi=-115.47^{\circ}$, using the linear relationship), this should be relatable to the differences in the surroundings, including counter-ion structure ${ }^{6}$ and possibly electrostatic field effects associated with neighboring regions in a charged coil. A more secure understanding of the spectrum, as provided by normal-mode analysis, obviously permits it to be used for a more rigorous interpretation of structure.

This research was supported by National Science Foundation grants DMB-8517812 and DMR-8303610.

\section{References}

1. Tiffany, M. L. \& Krimm, S. (1968) Biopolymers 6, 1379-1381.

2. Krimm, S. \& Mark, J. E. (1968) Proc. Natl. Acad. Sci. USA 60, 1122-1129.

3. Tiffany, M. L. \& Krimm, S. (1968) Biopolymers 6, 1767-1770.

4. Tiffany, M. L. \& Krimm, S. (1969) Biopolymers 8, 347-359.

5. Ronish, E. W. \& Krimm, S. (1972) Biopolymers 11, 1919-1928.

6. Krimm, S., Mark, J. E. \& Tiffany, M. L. (1969) Biopolymers 8, 695-697.

7. Tiffany, M. L. \& Krimm, S. (1972) Biopolymers 11, 2309-2316.

8. Tiffany, M. L. \& Krimm, S. (1973) Biopolymers 12, 575-587.

9. Krimm, S. \& Tiffany, M. L. (1974) Isr. J. Chem. 12, 189-200.

10. Rippon, W. B. \& Walton, A. G. (1971) Biopolymers 10, 1207-1212.

11. Rippon, W. B. \& Walton, A. G. (1972) J. Am. Chem. Soc. 94, 4319-4324.

12. Pal, M. K. \& Mandel, M. (1979) Biopolymers 18, 2267-2277.

13. Makarov, A. A., Esipova, N. G., Lobachev, V. M., Grishkovsky, B. A. \& Pankov, Y. A. (1984) Biopolymers 23, 5-22.

14. Srinivasan, R., Balasubramanian, B. \& Rajan, S. S. (1976) Science 194, 720-721.

15. Koenig, J. L. \& Frushour, B. (1972) Biopolymers 11, 1871-1892.

16. Yu, T.-J., Lippert, J. L. \& Peticolas, W. L. (1973) Biopolymers 12, 2161-2176.

17. Painter, P. C. \& Koenig, J. L. (1976) Biopolymers 15, 229-240.

18. Andries, J. C., Anderson, J. J. \& Walton, A. G. (1971) Biopolymers 10, 1049-1057.

19. Lotz, B. \& Keith, H. D. (1971) J. Mol. Biol. 61, 195-200.

20. Dwivedi, A. M. \& Krimm, S. (1982) Biopolymers 21, 2377-2397.

21. Sugawara, Y., Harada, J., Matsuura, H. \& Shimanouchi, T. (1978) Biopolymers 17, 1405-1421.

22. Krimm, S. \& Bandekar, J. (1986) Adv. Protein Chem. 38, 181-364.

23. Datye, V. K. \& Krimm, S. (1986) J. Chem. Phys. 84, 6989-6996.

24. Sengupta, P. K., Krimm, S. \& Hsu, S. L. (1984) Biopolymers 23, 1565-1594.

25. Sengupta, P. K. \& Krimm, S. (1985) Biopolymers 24, 1479-1491.

26. Sengupta, P. K. \& Krimm, S. (1985) Spectrochim. Acta 41 A, 205-207. 
27. Keith, H. D. (1970) Biopolymers 10, 1099-1101.

28. Hiltner, W. A., Hopfinger, A. J. \& Walton, A. G. (1972) J. Am. Chem. Soc. 94, 4324-4327. 29. Dwivedi, A. M. \& Krimm, S. (1982) Macromolecules 14, 186-193.

30. Dwivędi, A. M. \& Krimm, S. (1983) Macromolecules 16, 340.

31. Dwivedi, A. M. \& Krimm, S. (1984) Biopolymers 23, 923-943.

32. Keith, H. D., Giannoni, G. \& Padden, F. J. (1969) Biopolymers 7, 775-792.

33. Hsu, S. L. (1975) Ph.D. thesis, University of Michigan.

Received July 18, 1986

Accepted July 30, 1986 